\title{
Ursachen finden, im Team arbeiten, pneumologischen Nachwuchs fördern
}

\section{Blickdiagnosen \\ Welche Diagnose vermuten Sie? FOKUS}

Eine 18-jährige Patientin wurde stationär aufgenommen, da sie seit knapp einer Woche unter starken Kopfschmerzen, Photophobie und Nackensteife litt. Abgesehen von einer ambulant erworbenen, mit Amoxicillin behandelten Pneumonie ein Jahr zuvor war sie bis dahin gesund gewesen. In der Untersuchung zeigte sie sich akut krank mit den typischen Anzeichen einer Meningitis von Übelkeit und Erbrechen über Nackensteife bis hin zur Photophobie. Eine Lymphadenopathie war nicht zu erkennen. Die Thoraxuntersuchung ergab verminderten Lufteintritt und dumpfe Klopfgeräusche oben rechts. Alle anderen untersuchten Systeme waren unauffällig. Anhand eines positiven Kryptokokken-Latex-Antigentests des Liquor cerebrospinalis wurde eine Kryptokokkenmeningitis diagnostiziert. Alle Laborparameter einschließlich großem Blutbild und Serum-Harnstoff, -Kreatinin und -Elektrolyte lagen im Normbereich. Ein HIV-Test war negativ. Im Thoraxröntgen zeigte sich eine umfangreiche pleurale Raumforderung, die den Großteil des oberen rechten Bereichs einnahm (Abb. 1).

Die Behandlung der Meningitis erfolgte durch intravenöse Gabe von Amphotericin B 250 mg täglich für 3 Wochen, mit gutem klinischem Ansprechen. Ein erneutes Thoraxröntgen vor der Entlassung ergab keine Größenveränderung in der pleuralen Raumforderung. Ein Thorax-CT zeigte eine umfangreiche subpleurale Raumforderung im oberen rechten Flügel (Abb. 2). Wie lautet Ihre Diagnose? Welche Maßnahmen würden Sie ergreifen?

Übersetzung aus Respiration. 2003;70:655-657, DOl: 10.1159/000075216

\section{Auflösung unter:}

www.karger.com/blickdiagnose-kkp-5-2020

Kontakt: Dr. A.A.G. de Klerk, Department of Internal Medicine, US Faculty of Health Sciences, PO Box 19063, Tygerberg 7505, Südafrika, E-Mail adeklerk@ sun.ac.za

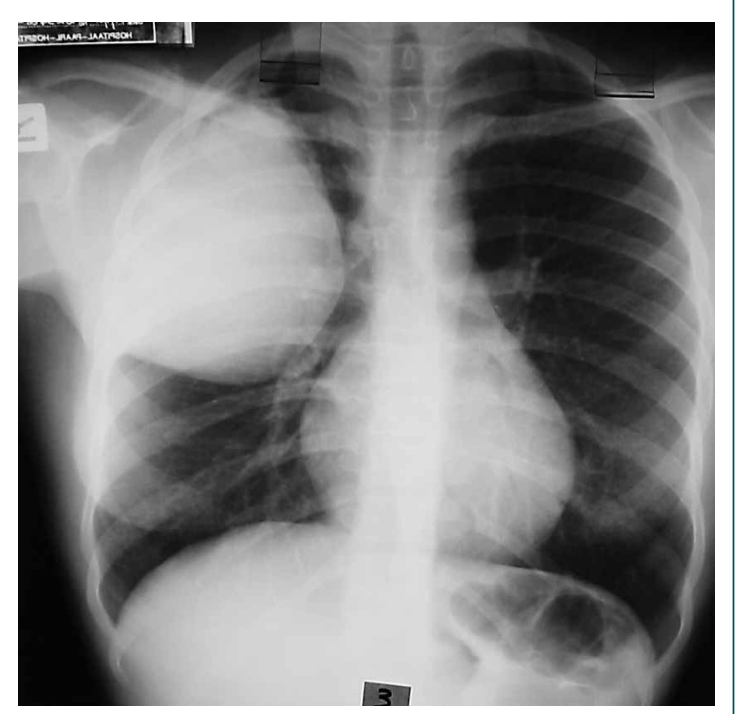

Abb. 1. Umfangreiche pleurale Raumforderung im Thoraxröntgen.

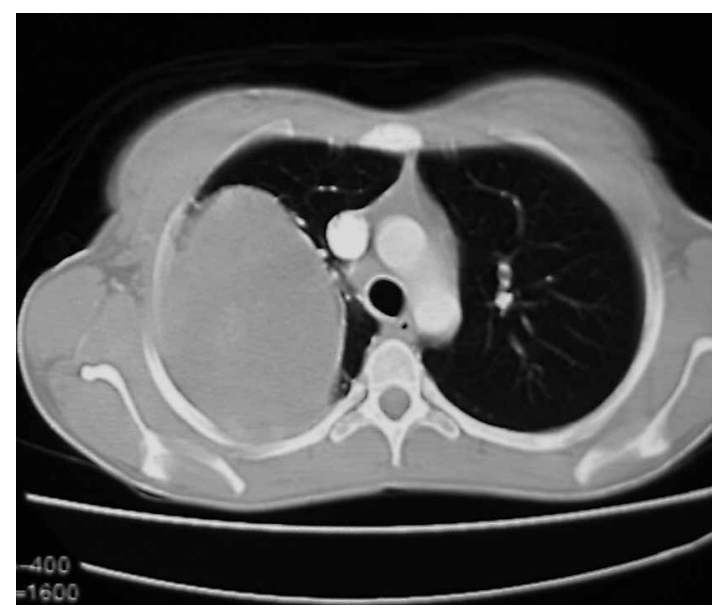

Abb. 2. Umfangreiche subpleurale Raumforderung. 


\section{Aus der Praxis}

\section{Der Umgang mit dem Pflegepersonal als Arzt}

Beginnt man seine Assistenz in einem Klinikum, trifft man oft auf sehr erfahrene Krankenschwestern und Krankenpfleger, die einem medizinische Tipps geben. Sollte man sie annehmen?

Um es vorwegzunehmen: Man kann die eingangs gestellte Frage nicht mit einem klaren «Ja» oder «Nein» beantworten, weil auch hier der Ton die Musik macht. Wenn eine erfahrene Schwester Vorschläge macht, was die Diagnose und Therapie von Patienten angeht, dann sollte man sich diese Vorschläge anhören. Pflegekräfte haben oft eine lange Berufserfahrung und die zunehmende Akademisierung ihrer Ausbildung macht den immer breiteren Erwerb von Fachwissen möglich. Dennoch bist du der Arzt oder die Ärztin und hast damit die Durchführungsverantwortung, während das Pflegepersonal in der Anordnungsverantwortung ist, das heißt du haftest später für die getroffene Entscheidung und nicht das Pflegepersonal. Wichtig ist deshalb, dass man Ratschläge anderer nicht vorschnell annimmt, sondern sich selbst in aller Ruhe ein Bild macht. Im Zweifel fragt man den Oberarzt oder den Weiterbildungsbevollmächtigten, ob die angedachte Therapie sinnvoll ist.

\section{Parallele Hierarchien}

Bei dem Pflegepersonal gibt es eine Pyramidenhierachie, die von der Hilfsschwester über die Schwester, zur Stationsschwester, zur Pflegedienstleitung bis zur Pflegedirektion reicht. Wie bei den Ärzten gibt es auch hier eine Weisungsbefugnis von oben nach unten. Grundsätzlich gilt: Die pflegerische Versorgung übernimmt das Pflegepersonal selbstständig, die medizinischen Aufgaben werden hingegen von dir als Arzt oder Ärztin delegiert, und zwar sowohl an die Hilfsschwester als auch an die Stationsschwester. vorzugehen. Aber manchmal kann auch das größte diplomatische Geschick keine direkte Auseinandersetzung verhindern. Zur Verdeutlichung ein Beispiel: Nehmen wir an, du beginnst deine Assistenz und die Stationsschwester, die erheblich älter ist, will von dir gesiezt werden, während sie dich mit «Du» anspricht. Auf sehr direkte Weise versucht sie also das Machtverhältnis umzukehren. Hier empfiehlt sich der direkte Weg der Kommunikation. Mach ihr deutlich, dass ihr euch in einem Dienstverhältnis befindet und du deshalb das höflichere «Sie» bevorzugst. Du wirst sie mit diesem Vorgehen nicht als Freundin gewinnen, aber sie hat es ja selbst auf die Konfrontation angelegt und ist damit eine schwierige Kollegin, mit der du ohnehin nicht warm geworden wärst.

Andererseits kann die Expertise des Fachpflegepersonals lebensentscheidend sein. In tragischer Weise verdeutlicht dies der Tod der Britin Elaine Bromiley. Sie verstarb aufgrund von Fehlern bei der Narkoseeinleitung, die teilweise vermeidbar gewesen wären, wenn die behandelnden Anästhesisten auf die Ratschläge der Anästhesieschwestern gehört hätten. Letztendlich geht es auf Station um Teamwork, bei der jeder sein Wissen einbringen dürfen sollte. Eine junge Ärztegeneration, die um ihre Verantwortung weiß, klare Grenzen zieht, aber auch gegenüber Kritik aufgeschlossen ist, kann in Zukunft sicherlich viel bewirken.

Quelle: Der Umgang mit dem Pflegepersonal als Arzt. Ein Beitrag auf www.operation-karriere.de (www.operation-karriere.de/karriereweg/bewerbung-berufsstart/der-umgang-mit-dem-pflegepersonalals-arzt.html)

\section{Konflikte mit dem Pflegepersonal: 2 Beispiele}

Es ist oft gar nicht so leicht, als «Frischling» einer Stationsschwester Aufgaben zu erteilen. Deshalb ist mit großem Fingerspitzengefühl

\section{OPERATION KARRIERE}

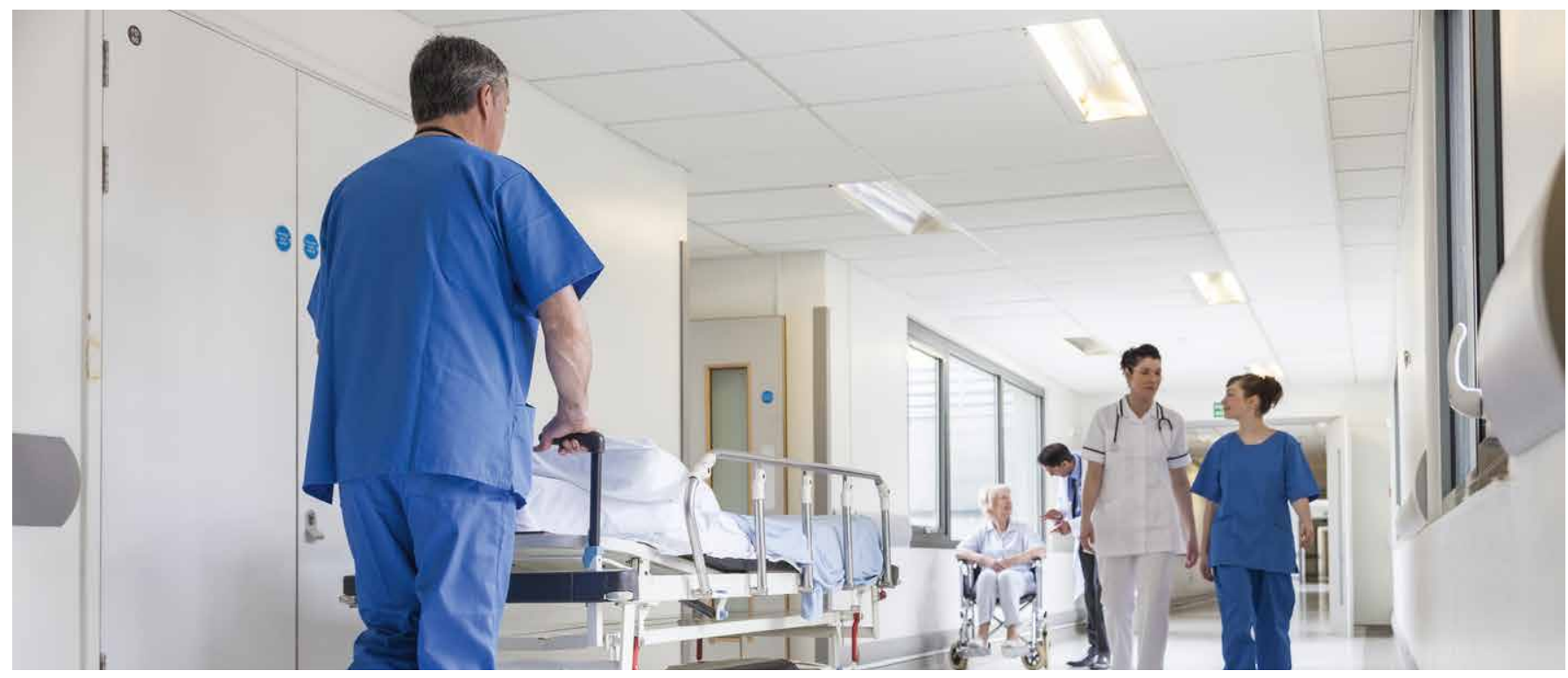

Duzt oder siezt man sich? Der Umgangston ist von Station zu Station unterschiedlich. @ Spotmatik/Shutterstock 


\section{Junge Ärztinnen und Ärzte}

\section{Die Arbeitsgruppe YoungDGP - Pneumologischer Nachwuchs in Deutschland}

Demographischer Wandel, rascher medizinischer Fortschritt, zunehmende Prozesse der Ökonomisierung und Digitalisierung im Gesundheitswesen, sowie die Veränderung gesellschaftlicher Normen in Bezug auf die Arbeitsplatzgestaltung und das Verhältnis von Beruf und Familie bzw. Freizeit wirken sich in unterschiedlicher Weise auf die Arbeits- und Weiterbildungsbedingungen des ärztlichen Nachwuchses aus. Gerade für den Arztberuf bedeutet der demographische Wandel nicht nur eine steigende Anzahl chronisch kranker, älterer Patienten, die versorgt und behandelt werden müssen, sondern auch einen unmittelbar bevorstehenden Generationswechsel innerhalb der Ärzteschaft. Auf den Prozess des demographischen Wandels lässt sich nur wenig Einfluss nehmen. Umso wichtiger ist es, junge Ärztinnen und Ärzte dauerhaft für die Tätigkeit in der direkten Patientenversorgung zu begeistern. Gute Arbeits- und Weiterbildungsbedingungen können verhindern, dass Ärzte in alternative Betätigungsfelder fern der Patientenversorgung «verloren gehen» oder Deutschland verlassen. Eine gute Vereinbarkeit von Familie und Beruf scheint dabei ein elementarer Baustein zu sein, um dem weiter steigenden Anteil an Frauen in der Medizin, sowie den generell veränderten Familien- und Lebenskonzepten zu begegnen. Die Facharztqualifikation ist sowohl im Krankenhaus als auch im ambulanten Sektor eine notwendige Voraussetzung, um sich beruflich weiterzuentwickeln oder sich um eine Niederlassung in selbstständiger, kassenärztlicher Tätigkeit bewerben zu können. Vor einer möglichen selbstständigen, aber in jedem Fall selbstverantwortlichen Tätigkeit steht eine Phase des fundierten Wissenserwerbs innerhalb der Facharztweiterbildung. Eine gut strukturierte Organisation der jeweiligen Weiterbildungsabschnitte, sowie regelmäßige Evaluation des persönlichen Fortschritts in Bezug auf Fähigkeiten, Fertigkeiten und Kenntnisse der Weiterzubildenden sind ist eine
Wichtigste Anliegen der AG YoungDGP

- bestmögliche ärztliche Aus-, Weiterbildungs- und Arbeitsbedingungen

- Förderung des wissenschaftlichen Nachwuchses

- enger Austausch mit anderen nationalen und internationalen Nachwuchsgruppen

- Vertretung des Nachwuchses gegenüber dem Vorstand der DGP, in den Sektionen und Arbeitsgruppen und anderen Projekten mit Nachwuchsbezug

notwendige und elementare Voraussetzung, um die jeweilige Weiterbildung in einer langfristig zufriedenstellenden Qualität zu absolvieren.

Die AG YoungDGP hat sich in der Folge des 60. Kongresses der Deutschen Gesellschaft für Pneumologie und Beatmungsmedizin e.V. (DGP) 2019 in München erstmals formiert, um die Belange angehender Pneumologinnen und Pneumologen innerhalb der Fachgesellschaft zu vertreten. Die AG YoungDGP setzt sich ein für bestmögliche ärztliche Aus-, Weiterbildungs- und Arbeitsbedingungen, für eine Förderung des wissenschaftlichen Nachwuchses, sowie für einen engen Austausch mit anderen nationalen und internationalen Nachwuchsgruppen. Die AG vertritt den Nachwuchs gegenüber dem Vorstand der DGP, in den Sektionen und Arbeitsgruppen, sowie bei Projekten, die einen Bezug zum Nachwuchs haben.

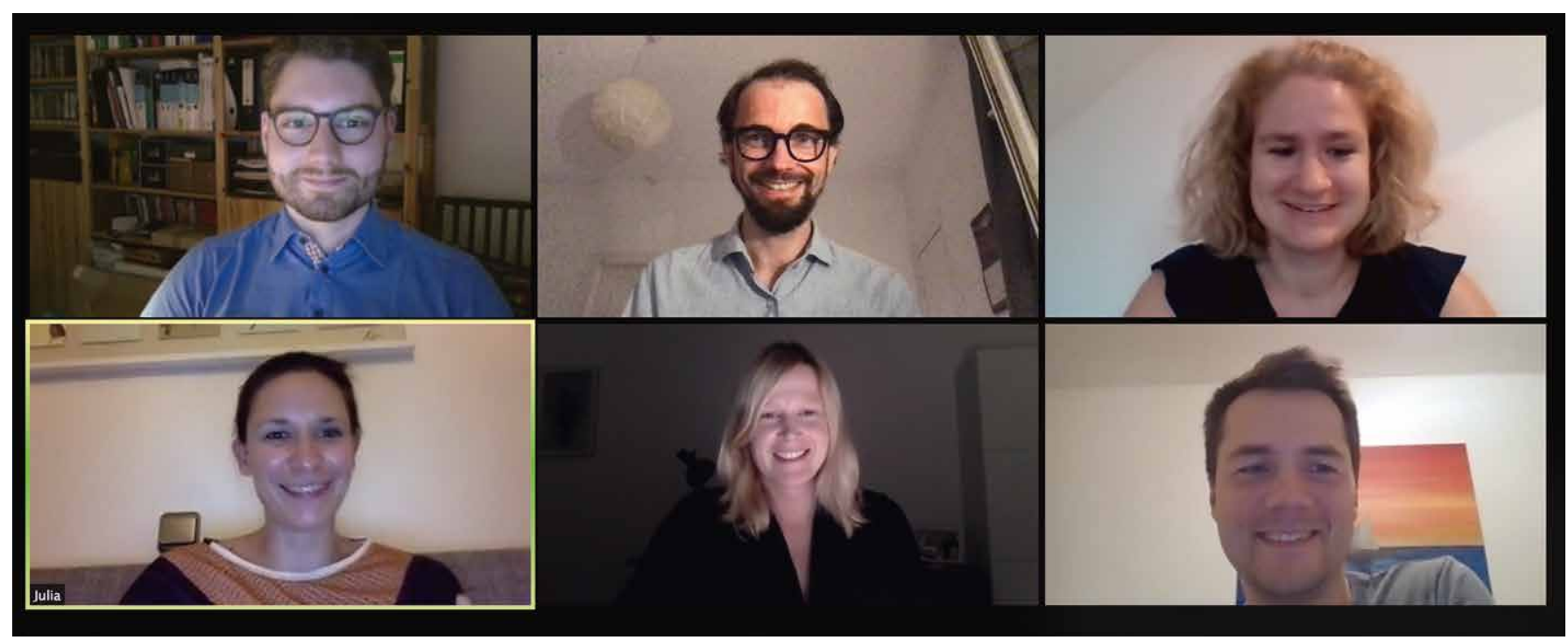

Videokonferenz der AG YoungDGP in Covid-19-Zeiten (von oben links nach unten rechts: Espen Groth, Prof. Dr. Thomas Bahmer, Dr. Myriam Koch, Dr. Julia Wälscher, Dr. Tina Schreiber, Dr. Christoph Fisser) 
Eines unserer Ziele ist es, die Weiterbildungsqualität und -zufriedenheit der angehenden Pneumologinnen und Pneumologen in Deutschland strukturiert zu erfassen und darauf aufbauend gemeinsam mit den Weiterbildungsbefugten, sowie Standesvertretern und Entscheidern im Gesundheitswesen die Qualität zu verbessern, bzw. an die sich in Veränderung befindlichen Rahmenbedingungen anzupassen. Mit Unterstützung der DGP und des Berufsverbands der Pneumologen, Schlaf- und Beatmungsmediziner in Deutschland (BdP) wurde eine Umfrage erstellt, die sich insbesondere an Ärztinnen und Ärzte in der Weiterbildung zum Facharzt für Innere Medizin, hier v.a. mit dem Schwerpunkt Pneumologie, richtet. Die Umfrage erfolgte von September bis November 2019 anonymisiert, internetbasiert und beleuchtete fünf übergeordnete Themenfelder:

- Arbeitsbedingungen im Berufsalltag,

- Organisation der ärztlichen Fort- und Weiterbildung,

- Vereinbarkeit von Beruf und Familie bzw. Freizeit,

- Einfluss ökonomischer Erwägungen auf ärztliches Handeln,

- sowie Vereinbarkeit von klinischem Arbeitsalltag mit Forschung und Wissenschaft.

Darüber hinaus wurde mit dem «Modell beruflicher Gratifikationskrisen» ein standardisiertes Messinstrument zur Erfassung der psychosozialen Arbeitsbelastung verwendet. Insgesamt konnten Fragebögen von 224 Weiterbildungsassistentinnen und -assistenten (w/m 54,5\%/45,5\%) ausgewertet werden, die sich im Schnitt in ihrem 5. Weiterbildungsjahr befanden und in $46,4 \%$ direkt den Facharzt (FA) für Innere Medizin und Pneumologie anstrebten, während 12,5\% zunächst den FA für Allgemeine Innere Medizin anstrebten und $35,7 \%$ sich aktuell in der Weiterbildung zum FA für
Pneumologie (zusätzlich zum FA für Innere Medizin) befanden. Die umfassenden Ergebnisse der Umfrage wurden erstmals auf dem Europäischen pneumologischen Kongress (ERS) im September 2020 digital vorgestellt.

Die AG YoungDGP freut sich über Interesse an ihrer Arbeit, eine Kontaktaufnahme ist unter youngdgp@pneumologie.de jederzeit unverbindlich und unkompliziert möglich!

\section{PNEUMOLOGIE ist Zukunft https://zukunft.pneumologie.de/ unterstützung_der_dgp/ag_youngdgp}

Autoren: Dr. Julia Wälscher (Universitätsmedizin Essen, Ruhrlandklinik, Klinik für Pneumologie, Zentrum für interstitielle und seltene Erkrankungen, Essen, Deutschland; Stellvertretende Sprecherin der AG YoungDGP), Dr. Christoph Fisser (Universitätsklinikum Regensburg, Klinik und Poliklinik für Innere Medizin II, Regensburg, Deutschland; Mitglied der AG YoungDGP), Dr. Tina Schreiber (Universitätsklinikum Bonn, Sektion für Pneumologie, Herzzentrum Bonn, Bonn, Deutschland; Mitglied der AG YoungDGP), Dr. Myriam Koch (Universitätsklinikum Regensburg, Klinik und Poliklinik für Innere Medizin II, Regensburg, Deutschland; Mitglied der AG YoungDGP), Dr. Matthias Raspe (Charité - Universitätsmedizin Berlin, corporate member of Freie Universität Berlin, Humboldt-Universität zu Berlin, and Berlin Institute of Health, Medizinische Klinik m.S. Infektiologie und Pneumologie, Charitéplatz 1, 10117 Berlin; Sprecher der AG Junge Internisten der DGIM und Mitglied der AG YoungDGP), Prof. Dr. Thomas Bahmer (Universitätsklinikum SchleswigHolstein, Campus Kiel, Medizinische Klinik I, und Christian-Albrechts-Universität zu Kiel, Kiel, Deutschland; Airway Research Center North (ARCN), Deutsches Zentrum für Lungenforschung (DZL), Sprecher der AG YoungDGP) 